\title{
Two-dimensional vortices in superconductors
}

\author{
BO CHEN ${ }^{1}$, W. P. HALPERIN ${ }^{*}$, PRASENJIT GUPTASARMA2 ${ }^{2}$, D. G. HINKS ${ }^{3}$, V. F. MITROVIĆ ${ }^{4}$, A. P. REYES ${ }^{5}$ \\ AND P. L. KUHNS 5 \\ ${ }^{1}$ Department of Physics and Astronomy, Northwestern University, Evanston, Illinois 60208, USA \\ ${ }^{2}$ Department of Physics, University of Wisconsin-Milwaukee, Wisconsin 53211, USA \\ ${ }^{3}$ Materials Science and Technology Division, Argonne National Laboratory, Argonne, Illinois 60439, USA \\ ${ }^{4}$ Department of Physics, Brown University, Providence, Rhode Island 02912, USA \\ ${ }^{5}$ National High Magnetic Field Laboratory Tallahassee, Florida 32310, USA \\ *e-mail: w-halperin@northwestern.edu
}

Published online: 11 February 2007; doi:10.1038/nphys540

Superconductors have two key characteristics: they expel magnetic field and they conduct electrical current with zero resistance. However, both properties are compromised in high magnetic fields, which can penetrate the material and create a mixed state of quantized vortices. The vortices move in response to an electrical current, dissipating energy and destroying the zero-resistance state ${ }^{1}$. One of the central problems for applications of high-temperature superconductivity is the stabilization of vortices to ensure zero electrical resistance. We find that vortices in the anisotropic superconductor $\mathrm{Bi}_{2} \mathrm{Sr}_{2} \mathrm{CaCu}_{2} \mathrm{O}_{8+\delta}(\mathrm{Bi}-2212)$ have a phase transition from a liquid state, which is inherently unstable, to a two-dimensional vortex solid. We show that at high field the transition temperature is independent of magnetic field, as was predicted theoretically for the melting of an ideal two-dimensional vortex lattice ${ }^{2,3}$. Our results indicate that the stable solid phase can be reached at any field, as may be necessary for applications involving superconducting magnets ${ }^{4-6}$. The vortex solid is disordered, as suggested by previous studies at lower fields ${ }^{7,8}$. But its evolution with increasing magnetic field exhibits unexpected threshold behaviour that needs further investigation.

Soon after the discovery of cuprate superconductivity, it was recognized that their high transition temperature $\left(T_{\mathrm{c}}\right)$ would mean that thermal fluctuations can produce a liquid vortex state ${ }^{9}$. In fact, the thermodynamic transition to superconductivity in a magnetic field occurs between a thermally fluctuating liquid vortex phase to one or more solid phases ${ }^{10}$. The liquid vortex state is inherently unstable with non-zero electrical resistance. For extremely anisotropic materials, such as Bi-2212, the liquid phase covers a wide range of temperature; but it is not known exactly how wide this is in high magnetic fields. For fields perpendicular to the conducting planes, $H \| c$-axis, the transition temperature between liquid and solid vortex phases, $T_{\mathrm{m}}(H)$, is principally controlled by vortex-vortex interactions that get stronger as the density of vortices increases, proportional to the field. The supercurrents that form each vortex are mainly confined to the conducting planes and, in high field, they lose their coherence from one plane to the next so that vortices are expected to become two dimensional. It is remarkable that the theory $y^{3}$ for the two-dimensional vortex

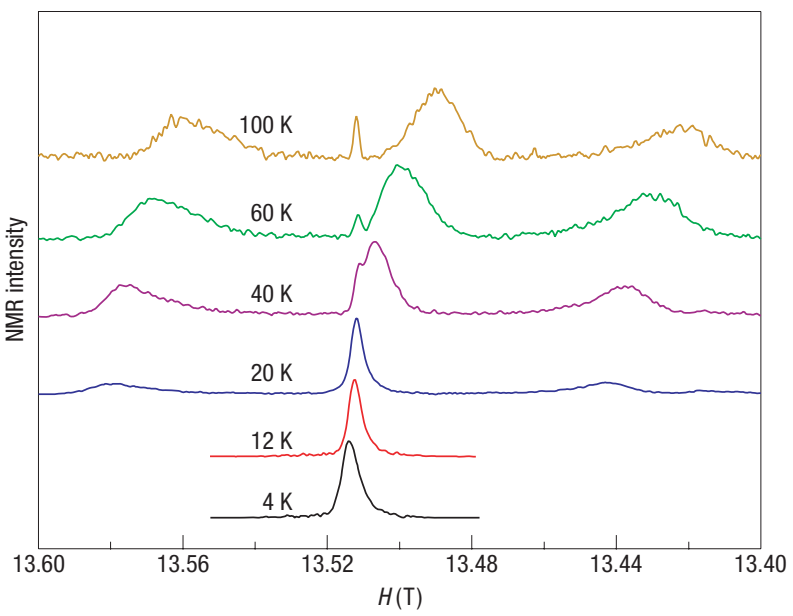

Figure 1 Spectra of ${ }^{17} \mathrm{O} \mathrm{NMR}$ in Bi-2212 for magnetic field parallel to the $c$ axis at fixed frequency. The sample is an overdoped, $28 \mathrm{mg}$, single crystal of $\mathrm{Bi}_{2} \mathrm{Sr}_{2} \mathrm{CaCu}_{2} \mathrm{O}_{8+\delta}$ (Bi-2212), with $T_{\mathrm{c}}=75 \mathrm{~K}$ and with $\approx 60 \%$ of the ${ }^{16} 0$ exchanged for ${ }^{17} 0$. The optimally doped $T_{\mathrm{c}}=93 \mathrm{~K}$. In field-sweep experiments ( $T=20,40,60$, $100 \mathrm{~K}$ ), a decreasing Knight shift moves the NMR spectrum to the left. At high temperatures, two oxygen sites can be distinguished. The central transition, $\langle-(1 / 2) \leftrightarrow+(1 / 2)\rangle$ for oxygen in the copper-oxygen plane, $0(1)$, is the wide line near $13.49 \mathrm{~T}$ at $100 \mathrm{~K}$. The central transition for the oxygen in the strontium-oxygen plane, $0(2)$, is the narrow line near the zero Knight shift position at $13.51 \mathrm{~T}$. The other two peaks are quadrupolar satellites of the $0(1)$ resonance. For $T=4$ and $12 \mathrm{~K}$, we show the Fourier transform of the echo at fixed field, having a smaller spectral bandwidth so that satellites are not observed here. Our separate measurement of the satellite intensity at $T / T_{\mathrm{m}}=0.67$ compared with the central transition is the same as that at $T / T_{\mathrm{m}}=1.33$, confirming this picture. The spectra at different temperatures are normalized to have the same peak intensity of the $0(1)$ central transition.

melting transition has only one parameter, the magnetic field penetration depth, and that this simple picture has not yet been experimentally confirmed. 


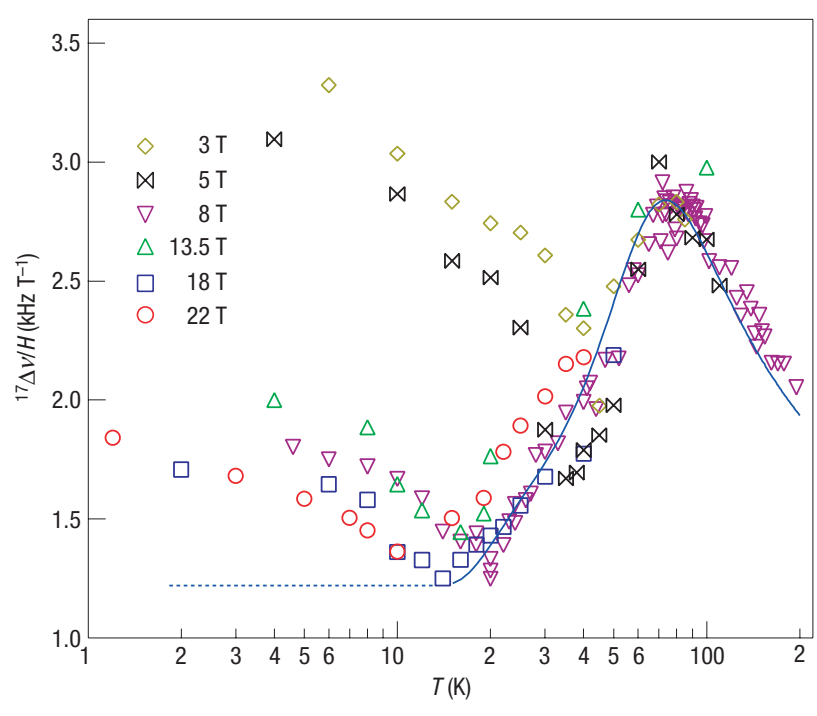

Figure 2 Temperature dependence of the $\mathbf{0}(\mathbf{1})$ linewidth. Temperature dependence of the linewidth of the ${ }^{17} 0$ NMR central transition presented as the square root of the second moment of the frequency spectrum, ${ }^{17} \Delta v$, divided by magnetic field, $H$, in the range 3 to $22 \mathrm{~T}$. Fields above $8 \mathrm{~T}$ were obtained at the National High Magnetic Field Laboratory in Tallahassee, Florida. Measurements at 27 and $29 \mathrm{~T}$ are included in Figs 3 and 4, but omitted here for clarity. On decreasing the temperature, there is a common behaviour independent of magnetic field, represented by the smooth curve and its continuation to low temperature (dotted curve), that can be understood in terms of defects in the copper-oxygen plane induced by oxygen doping. This curve is given by $\Delta v=\Delta v_{0}+K H D / T$, where $\Delta v_{0}$ is a background magnetic contribution to the linewidth possibly associated with regions of the sample where superconductivity is suppressed, $K(T)$ is the measured Knight shift, $H / T$ is the field-to-temperature ratio and $D$ is a Curie constant. The key feature of the data is the systematic break from this curve that we identify with the transition from a liquid to a solid vortex state.

We use nuclear magnetic resonance (NMR) of ${ }^{17} \mathrm{O}$ to detect the melting transition of vortices as a function of temperature and magnetic field. NMR can be carried out on selected elements in site-specific locations in the structure ${ }^{11,12}$ as can be seen in the ${ }^{17} \mathrm{O}$ spectra in Fig. 1. There are three stoichiometric positions for oxygen in $\mathrm{Bi}-2212$ : in atomic planes containing $\mathrm{Cu}$, the $\mathrm{O}(1)$ site; Sr planes, the $\mathrm{O}(2)$ site; and $\mathrm{Bi}$ planes. The last oxygen atoms are unobserved ${ }^{11}$ owing to disorder in this plane. In addition, there is a small amount of non-stoichiometric oxygen, $\delta$-oxygen, too small to be observed directly by NMR and whose location in the structure is not yet established ${ }^{13}$. The parts of the NMR spectra in the middle range of field in Fig. 1 are the central transitions from the $\mathrm{O}(1)$ (broad) and $\mathrm{O}(2)$ (narrow) sites. The electronic coupling to ${ }^{17} \mathrm{O}$ is much stronger for $\mathrm{O}(1)$ compared with $\mathrm{O}(2)$, as is apparent from the temperature dependences in Fig. 1. This is confirmed by measurements of the spin-lattice relaxation rate, which are one order of magnitude larger for $\mathrm{O}(1)$ compared with $\mathrm{O}(2)$. The narrowness of the $\mathrm{O}(2)$ resonance indicates a homogeneous electronic environment with negligible spin shift (Knight shift) and uniform electric field gradient. For fast pulse repetition, as is the case for our measurements in Fig. 1, $\mathrm{O}(2)$ is partially saturated and comprises less than $6 \%$ of the spectrum at $60 \mathrm{~K}$, decreasing with decreasing temperature to $2 \%$ at $40 \mathrm{~K}$. We remove it numerically in this range of temperature and below this, it has negligible contribution to the spectrum. The $\mathrm{O}(2)$ resonance serves as a useful marker for the zero spin-shift position of ${ }^{17} \mathrm{O}$ for both $\mathrm{O}(1)$ and
$\mathrm{O}(2)$ sites. Here, we focus on ${ }^{17} \mathrm{O}(1) \mathrm{NMR}$ as a probe of the local magnetic field in the $\mathrm{CuO}_{2}$ plane.

Decreasing the temperature below the superconducting transition temperature, we find that the ${ }^{17} \mathrm{O}(1)$ resonance peaks move to the left, that is, to a higher field at a fixed NMR frequency, (lower frequency at fixed field) approaching the zero spin-shift position. Simultaneously, the central NMR line narrows. The decrease of the Knight shift to zero in the superconducting state is a characteristic signature for spin-singlet pairing. The line broadening with decreasing temperature in the normal state, Figs 1 and 2, can be associated with a Knight shift distribution introduced by the $\delta$-oxygen. Similar behaviour has been observed ${ }^{14}$ for chemical impurities, such as $\mathrm{Ni}, \mathrm{Zn}$ or $\mathrm{Li}$, substituted for $\mathrm{Cu}$ in the $\mathrm{CuO}_{2}$ plane in yttrium-barium-copper oxide (YBCO), and which form a local moment. Their contribution to the ${ }^{17} \mathrm{O} N M R$ linewidth is given by a Curie law, proportional to the ratio of applied magnetic field to temperature. In addition, we find that below $T_{\mathrm{c}}$ this linewidth is proportional to the temperature-dependent Knight shift, which we measure independently, thereby accounting for the decrease with decreasing temperature in Fig. 2 as shown by the smooth curve, assuming a temperature-independent residual contribution of $1.2 \mathrm{kHz} \mathrm{T}^{-1}$. In this region, liquid vortex dynamics effectively average to zero their associated local magnetic fields.

On further cooling in the superconducting state there is a sharp onset for a new contribution to the linewidth that is not proportional to applied magnetic field. The well-defined temperature at which this line broadening occurs is shown in Fig. 3, decreasing, but progressively more slowly, with increasing magnetic field in our range between 3 and $29 \mathrm{~T}$. We identify this behaviour with the formation of a solid vortex structure. More precisely, the extra linewidth corresponds to an inhomogeneous magnetic field distribution that is static on the NMR timescale, $\approx 0.1 \mathrm{~ms}$, and is asymmetric; see the $T=4 \mathrm{~K}$ spectrum in Fig. 1 . This behaviour is characteristic of the transition from liquid to solid vortex matter such as has been observed by muon spin relaxation ${ }^{7}$ $(\mu \mathrm{SR})$ and small-angle neutron scattering ${ }^{8}$ (SANS) in Bi-2212, and NMR in YBCO (ref. 15). Our observations are qualitatively consistent with extension to high magnetic fields of the vortex melting phase diagram, $H<0.1 \mathrm{~T}$, determined by Hall probe ${ }^{16,17}$ and transport measurements ${ }^{18}$. Khaykovich et al. ${ }^{16}$ explore this behaviour as a function of oxygen doping (anisotropy). NMR is complementary to these methods with definite advantages for detecting vortex melting at very high magnetic fields. The NMR or $\mu$ SR spectrum is a direct map of the magnetic field distribution from vortex supercurrents. Specifically, NMR with ${ }^{17} \mathrm{O}$ affords excellent resolution as a magnetometer on the atomic scale and has been exploited in previous work ${ }^{19}$ to spatially resolve and study excitations in the vortex core.

The strong upward curvature of the phase diagram in Fig. 3 has been anticipated theoretically ${ }^{3,10}$. Torque magnetometry in fields $H \leq 5 \mathrm{~T}$ gave similar indications ${ }^{20}$. For highly anisotropic superconductors, the electromagnetic interaction between vortices dominates the Josephson coupling between planes. In this high field limit, the simplest picture for vortex melting is a first-order thermodynamic transition ${ }^{3}$ given by

$$
T_{\mathrm{m}}(H)=T_{\mathrm{m}}^{2 \mathrm{D}}\left[1+\frac{b^{1 / v}}{\ln ^{1 / v}\left(H / H_{\mathrm{cr}}\right)}\right],
$$

for $H$ larger than a crossover field,

$$
H_{\mathrm{cr}} \approx \frac{2 \pi \phi_{0}}{\gamma^{2} d^{2}} \ln \left(\frac{\gamma d}{\xi_{a b}} \sqrt{1+\frac{\left(4 \pi \lambda_{\mathrm{c}}\right)^{2} d}{2\left(\phi_{0} \xi_{a b}\right)^{2}} k_{\mathrm{B}} T}\right) .
$$


The limiting two-dimensional melting temperature in equation (1) is

$$
T_{\mathrm{m}}^{2 \mathrm{D}}=A \frac{\phi_{0}^{2} d}{8 \pi \sqrt{3} k_{\mathrm{B}}\left(4 \pi \lambda_{a b}\right)^{2}},
$$

where $0.605 \leq A \leq 0.615$ comes from numerical calculations (A. E. Koshelev, private communication). This spread in $A$ reflects the existence of an intermediate phase because the ideal twodimensional melting scenario proceeds in two steps with an intervening hexatic phase bounded by continuous transitions ${ }^{10}$. The melting temperature, equation (3), depends on the layer spacing $^{21} d=1.5 \mathrm{~nm}$, the flux quantum $\phi_{0}$, Boltzmann's constant $k_{\mathrm{B}}$ and the single superconductive parameter $\lambda_{a b}$, which is the penetration depth for supercurrents in the $\mathrm{CuO}_{2}$ plane. The crossover field, $H_{\mathrm{cr}}$, depends mainly on the product of $d$ and the mass anisotropy factor, $\gamma=\lambda_{c} / \lambda_{a b}=\xi_{a b} / \xi_{c}$. Out-of-plane components are denoted by a subscript $c$ and $\xi$ is the coherence length. The numerical constants in the theory, equation (1), are $b \approx 1$ and the exponent $v=0.37$.

The NMR vortex lineshape is asymmetric but less so than for a perfect line-vortex lattice suggesting that the vortex structure is somewhat disordered. In fact, $\mu$ SR and SANS show that at low temperature and $H>0.1 \mathrm{~T}$, it is a vortex glass. We will assume that the difference in energy between disordered and perfect vortex structures, at least in high magnetic field, is small compared with the energy for condensation from liquid to solid, and we use the framework of equations (1)-(3) to analyse the freezing that we have observed. From a fit to the data, dashed curve in Fig. 3, we find the crossover field $H_{\mathrm{cr}}=2.5 \mathrm{~T}$ and $\gamma \approx 78$ and the two-dimensional melting transition temperature $T_{\mathrm{m}}^{2 \mathrm{D}}=12 \pm 1 \mathrm{~K}$ for which the penetration depth is $\lambda_{a b}=220 \pm 10 \mathrm{~nm}$. Precision measurements of the absolute value of the penetration depth at low temperature are notoriously difficult. From earlier reports for $\mathrm{Bi}-2212, \lambda_{a b}$ is $\approx 210 \mathrm{~nm}$ from magnetization data ${ }^{21}, 269 \pm 15 \mathrm{~nm}$ from cavity resonance methods $\mathrm{s}^{22}$ and $\approx 180 \mathrm{~nm}$ from $\mu$ SR (ref. 7 ). These results are in agreement with what we report here. From torque measurements, Iye et al. ${ }^{23}$ found $\gamma \geq 200$ for nearly optimally doped samples, and Watauchi et al. ${ }^{24}$ obtained a value of 91 on overdoped material using resistivity methods. Although $\gamma \approx 78$ is in this range, it must be considered approximate because the theory is imprecise in the crossover field region. If we constrain $\gamma$, to any value over this wide range, our fit for $T_{\mathrm{m}}^{2 \mathrm{D}}$ is unaffected. For a less overdoped crystal, $T_{\mathrm{c}}=85 \mathrm{~K}$, we find a slightly higher value, $T_{\mathrm{m}}^{2 \mathrm{D}}=16 \mathrm{~K}$.

Experiments consistent with the two-dimensional melting theory have basic significance. But they are also relevant to applications of superconductivity at very high magnetic field. One of the most promising materials for magnet wire ${ }^{4,5}$ to make a $30 \mathrm{~T}$ superconducting NMR magnet, as was recently recommended by the National Research Council ${ }^{6}$, is Bi-2212. To achieve this goal, there will be an upper limit on the operational temperature of the magnet, determined by supercurrents that are more easily stabilized in the vortex solid phase.

At low magnetic fields, $H \ll H_{\text {cr }}$, it is well established that there is a complex phase diagram for $\mathrm{Bi}-2212$ with transitions on cooling from liquid vortex matter to a solid. At low temperature, an increasing magnetic field drives a transition at $H^{*} \approx 0.1 \mathrm{~T}$ from Bragg glass ${ }^{25}$ to vortex glass, indicated by an abrupt increase in the symmetry of the $\mu$ SR spectrum ${ }^{7}$, a decrease in its second moment ${ }^{7}$ and the disappearance of Bragg peaks in SANS (ref. 8). At this transition, vortices lose coherence between planes. The resulting destructive interference between vortices on adjacent planes averages out the magnetic field distribution ${ }^{26-28}$ that is expected for straight line vortices such as those seen in the Bragg glass phase $^{7}$. This interference reduces both asymmetry and linewidth in

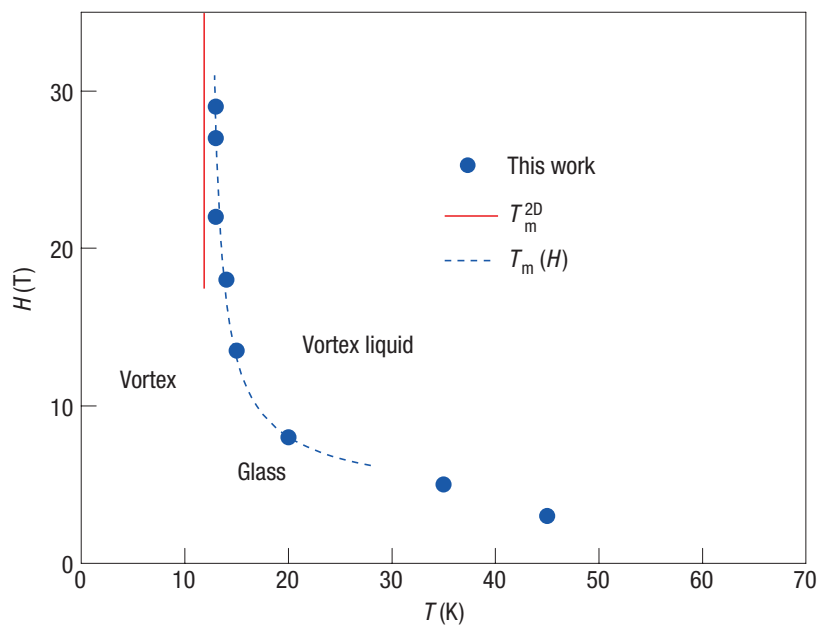

Figure 3 The magnetic-field-temperature phase diagram for vortex melting in Bi-2212 for $\boldsymbol{H} \| \boldsymbol{c}$. The transition temperatures, $T_{\mathrm{m}}(H)$, are identified from the data, as shown in Fig. 2. The two-dimensional vortex melting transition temperature, $T_{\mathrm{m}}^{2 \mathrm{D}}$ (vertical dashed line), is determined from a fit to the data. Deviations of the fit from the data are expected ${ }^{3}$ for $H \approx H_{\text {cr }}$.
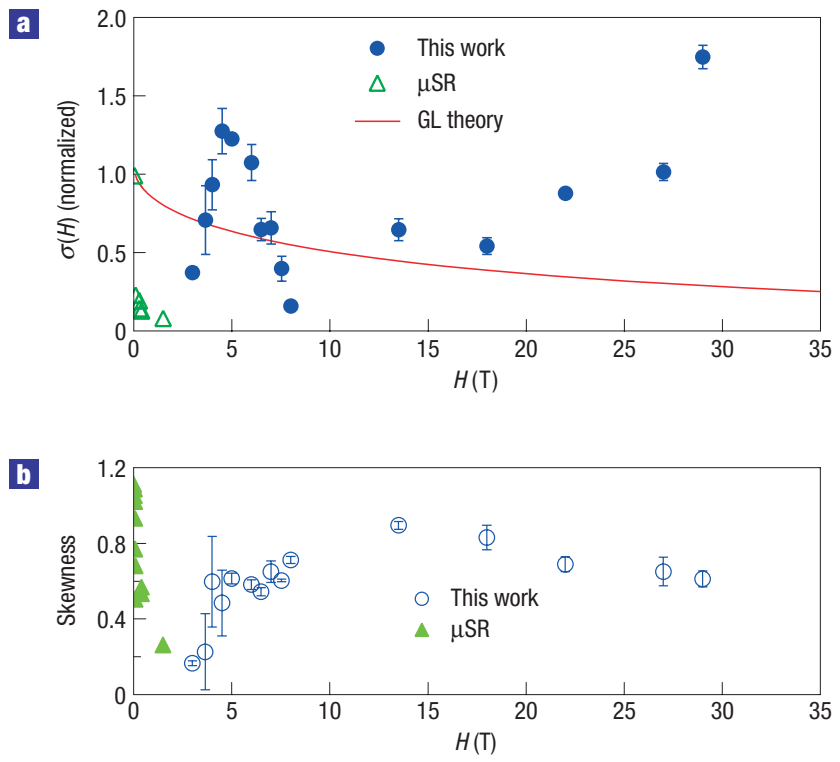

Figure 4 Magnetic field dependence of the vortex linewidth and asymmetry. a, The vortex contribution to the second moment of the NMR spectra, $\sigma(H)$, at low temperature, $T \approx 4 \mathrm{~K}$, given as the difference between the data shown in Fig. 3 and the background contribution, smooth curve in Fig. 2, expressed as a second moment. A comparison with that expected from rigid-line vortices is calculated from GL theory ${ }^{30}$, taking the upper critical field to be $230 \mathrm{~T}$. Low-field $\mu \mathrm{SR}$ measurements ${ }^{26,27}$ up to $1.5 \mathrm{~T}$ are shown as triangles with the lowest field less than $H^{*}$ in the Bragg glass phase, consistent with three-dimensional ordering there, but dropping dramatically in the vortex glass phase. The linewidth data are normalized to the theoretical value at low field. $\mathbf{b}$, The skewness, $\alpha=\left\langle\left[v-v_{1}\right]^{3}\right\rangle^{1 / 3} /$ $\left\langle\left[v-v_{1}\right]^{2}\right\rangle^{1 / 2}$, where $v_{1}$ is the first moment of the spectrum and the average is over the spectrum. A decrease in the second moment $H \approx 5 \mathrm{~T}$ with no drop in asymmetry suggests intraplanar vortex ordering. The error bars are statistical, calculated from the NMR spectra. 
the $\mu$ SR spectrum. With even larger fields, the second moment of the ${ }^{17} \mathrm{O}$ NMR spectrum, $\sigma(H)$, ( $\approx$ linewidth squared) progressively increases and asymmetry is restored, until the spectrum linewidth collapses at $5 \mathrm{~T}$. There are two possibilities for this anomalous behaviour at $5 \mathrm{~T}$. Either the transition is field-induced ordering (ordering in-plane) or field-induced disordering (further disorder between planes). The significant asymmetry in the spectrum in the high field phase compared with that at our lowest fields favours vortex ordering with increasing field. This scenario might follow if interplanar coupling is weakened with increasing field compared with intraplanar interactions, reducing frustration that originated from vortices in adjacent planes. As a consequence, vortices order two dimensionally and we observe that the second moment decreases abruptly. At much larger fields, we can see that the two-dimensional vortex solid becomes progressively more disordered. On theoretical grounds ${ }^{29}$, arbitrarily small amounts of impurity will lead to disorder of the vortex lattice. Sensitivity to quenched-in disorder should increase progressively with increasing magnetic field as the lattice spacing decreases with fixed vortex size.

To make a comparison with straight line vortices, we have carried out a Ginzburg-Landau (GL) calculation $^{30}$ of the corresponding magnetic field distribution, Fig. 4. Our measurement is of a similar magnitude as expected from this calculation, although the GL approximation does not capture the details of the observed field dependence. In fact, the transition from a liquid to a solid in high magnetic fields is predicted to occur first to a supersolid phase and then at lower temperatures to a decoupled solid phase where defects in the vortex lattice become zero dimensional ${ }^{10,31}$. NMR techniques may be helpful in exploring these fascinating aspects of vortex behaviour in strongly anisotropic superconductors.

\section{Received 23 August 2006; accepted 8 January 2007; published 11 February 2007.}

\section{References}

1. Anderson, P. W. \& Kim, Y. B. Hard superconductivity-theory of motion of Abrikosov flux lines. Rev. Mod. Phys. 36, 39-43 (1964).

2. Fisher, D. S. Flux-lattice melting in thin-film superconductors. Phys. Rev. B 22, 1190-1199 (1980).

3. Glazman, L. I. \& Koshelev, A. E. Thermal fluctuations and phase-transitions in the vortex state of a layered superconductor. Phys. Rev. B 43, 2835-2843 (1991).

4. Hasegawa, T. et al. 12 kA HTS Rutherford cable. IEEE Trans. Appl. Supercond. 14, 1066-1069 (2004)

5. Scanlan, R. M., Malozemoff, A. P. \& Larbalestier, D. C. Superconducting materials for large scale applications. Proc. IEEE 92, 1639-1654 (2004).

6. Opportunities in High Magnetic Field Science (The National Academies Press, Washington DC, 2005).

7. Lee, S. L. et al. Evidence for flux-lattice melting and a dimensional crossover in single-crystal $\mathrm{Bi}_{2.15} \mathrm{Sr}_{1.85} \mathrm{CaCu}_{2} \mathrm{O}_{8+\delta}$ from muon spin rotation studies. Phys. Rev. Lett. 71, 3862-3865 (1993).

8. Cubitt, R. et al. Direct observation of magnetic flux lattice melting and decomposition in the high- $T_{c}$ superconductor $\mathrm{Bi}_{215} \mathrm{Sr}_{195} \mathrm{CaCu}_{2} \mathrm{O}_{8+x}$. Nature 365, 407-411 (1993).

9. Nelson, D. R. Vortex entanglement in high- $T_{\mathrm{c}}$ superconductors. Phys. Rev. Lett. 60, 1973-1976 (1988).
10. Blatter, G., Feigel'man, M. V., Geshkenbein, V. B., Larkin, A. I. \& Vinokur, V. M. Vortices in high-temperature superconductors. Rev. Mod. Phys. 66, 1125-1388 (1994).

11. Trokiner, A. et al. ${ }^{17} \mathrm{O}$ NMR spectroscopy of $\mathrm{Bi}_{2} \mathrm{Sr}_{2} \mathrm{CaCu}_{2} \mathrm{O}_{8+x}$ high- $T_{\mathrm{c}}$ superconductor. Phys. Rev. $B$ 41, 9570-9573 (1990)

12. Takigawa, M. \& Mitzi, D. B. NMR studies of spin excitations in superconducting $\mathrm{Bi}_{2} \mathrm{Sr}_{2} \mathrm{CaCu}_{2} \mathrm{O}_{8+s}$ single crystals. Phys. Rev. Lett. 73, 1287-1290 (1994).

13. McElroy, K. et al. Atomic-scale sources and mechanism of nanoscale electronic disorder in $\mathrm{Bi}_{2} \mathrm{Sr}_{2} \mathrm{CaCu}_{2} \mathrm{O}_{8+\delta}$. Science 309, 1048-1052 (2005).

14. Bobroff, J. et al. Using Ni substitution and ${ }^{17} \mathrm{O}$ NMR to probe the susceptibility $\chi^{\prime}(\mathrm{q})$ in cuprates. Phys. Rev. Lett. 79, 2117-2120 (1997).

15. Reyes, A. P. et al. Vortex melting in polycrystalline $\mathrm{YBa}_{2} \mathrm{Cu}_{3} \mathrm{O}_{7}$ from ${ }^{17} \mathrm{O}$ NMR. Phys. Rev. B 55, 14737-14740 (1997).

16. Khaykovich, B. et al. Vortex-lattice phase transitions in $\mathrm{Bi}_{2} \mathrm{Sr}_{2} \mathrm{CaCu}_{2} \mathrm{O}_{8}$ crystals with different oxygen stoichiometry. Phys. Rev. Lett. 76, 2555-2558 (1996).

17. Fuchs, D. T. et al. Possible new vortex matter phases in $\mathrm{Bi}_{2} \mathrm{Sr}_{2} \mathrm{CaCu}_{2} \mathrm{O}_{8}$. Phys. Rev. Lett. 80, 4971-4974 (1998).

18. Torres, J. H. S., da Silva, R. R., Moehlecke, S. \& Kopelevich, Y. Vortex lattice in $\mathrm{Bi}_{2} \mathrm{Sr}_{2} \mathrm{CaCu}_{2} \mathrm{O}_{8}$ well above the first-order phase transition boundary. Physica C 408-410, 566-567 (2004).

19. Mitrović, V. F. et al. Spatially resolved electronic structure inside and outside the vortex cores of a high-temperature superconductor. Nature 413, 501-504 (2001).

20. Gammel, P. L., Schneemeyer, L. F., Wasczak, J. V. \& Bishop, D. J. Evidence from mechanical measurements for flux-lattice melting in single-crystal $\mathrm{YBa}_{2} \mathrm{Cu}_{3} \mathrm{O}_{7}$ and $\mathrm{Bi}_{2.2} \mathrm{Sr}_{2} \mathrm{Ca}_{0.8} \mathrm{Cu}_{2} \mathrm{O}_{8}$. Phys. Rev Lett. 61, 1666-1669 (1988)

21. Kogan, V. G., Ledveij, M., Simonov, A. Yu., Cho, J. H. \& Johnston, D. C. Role of vortex fluctuations in determining superconducting parameters from magnetization data for layered superconductors. Phys. Rev. Lett. 70, 1870-1873 (1993).

22. Prozorov, R. et al. Measurements of the absolute value of the penetration depth in high- $T_{c}$ superconductors using a low- $T_{\mathrm{c}}$ superconductive coating. Appl. Phys. Lett. 77, $4202-4204$ (2000).

23. Iye, Y. et al. Anisotropy of $\mathrm{Bi}_{2} \mathrm{Sr}_{2} \mathrm{CaCu}_{2} \mathrm{O}_{8+\delta}$ revisited. Physica C 199, 154-160 (1992).

24. Watauchi, S., Ikuta, H., Kobayashi, H., Shimoyama, J. \& Kishio, K. First-order vortex phase transition in $\mathrm{Bi}_{2} \mathrm{Sr}_{2} \mathrm{CaCu}_{2} \mathrm{O}_{y}$ single crystals with different carrier concentrations studied by resistivity measurements. Phys. Rev. B 64, 064520 (2001).

25. Klein, T. et al. A Bragg glass phase in the vortex lattice of a type II superconductor. Nature $\mathbf{4 1 3}$ 404-406 (2001).

26. Inui, M. \& Harshman, D. R. Muon-spin relaxation and magnetic flux lines in the mixed-state high temperature superconductors. Phys. Rev. B 47, 12205-12213 (1993).

27. Aegerter, C. M. et al. Angular dependence of the disorder crossover in the vortex lattice of $\mathrm{Bi}_{2.15} \mathrm{Sr}_{1.85} \mathrm{CaCu}_{2} \mathrm{O}_{8+\delta}$ by muon spin rotation and torque magnetometry. Phys. Rev. B 57, $1253-1258$ (1998).

28. Brandt, E. H. Magnetic-field variance in layered superconductors. Phys. Rev. Lett. 66, 3213-3216 (1991).

29. Larkin, A. I. \& Ovchinnikov, Yu. N. J. Low Temp. Phys. 34, 409-428 (1979).

30. Brandt, E. H. Properties of the ideal Ginzburg-Landau vortex lattice. Phys. Rev. B 68, 054506 (2003).

31. Crabtree, G. W. \& Nelson, D. R. Vortex physics in high-temperature superconductors. Phys. Today 38-45 (April 1997).

Acknowledgements

We gratefully acknowledge discussions with L. I. Glazman, A. E. Koshelev, D. C. Larbalastier, J. A. Sauls and E. Zeldov and contributions from E. E. Sigmund and P. Sengupta. We are grateful to Robert Smith for a detailed study of the satellites of $\mathrm{O}(1)$ in the vortex solid state. This work was supported by the Department of Energy, grant DE-FG02-05ER46248, and was carried out in part at the National High Magnetic Field Laboratory supported by the National Science Foundation and the State of Florida.

P.G. acknowledges support from the National Science Foundation, grant DMR 0449969. Correspondence and requests for materials should be addressed to W.P.H.

Author contributions

Experimental work and analysis was carried out by B.C., W.P.H., V.F.M., A.P.R. and P.L.K. Samples were prepared by P.G. and D.G.H.

Competing financial interests

The authors declare no competing financial interests.

Reprints and permission information is available online at http://npg.nature.com/reprintsandpermissions/ 